\title{
Experience with Cidofovir as an adjunctive therapy in a patient of adenovirus-induced macrophage activation syndrome in systemic arthritis
}

\author{
Priyankar Pal $^{1}$ (D) $\cdot$ Niladri Bose $^{2} \cdot$ Avishek Poddar $^{3} \cdot$ Kaustabh Chowdhury $^{3} \cdot$ Agnisekhar Saha $^{3}$
}

Received: 13 March 2020 / Revised: 17 April 2020 / Accepted: 23 April 2020 / Published online: 17 May 2020

(C) International League of Associations for Rheumatology (ILAR) 2020

\begin{abstract}
A 5-year-old female child, with known systemic juvenile idiopathic arthritis diagnosed at 18 months of age (on low dose Prednisolone + Methotrexate + Leflunomide + Tocilizumab), presented with fever for 1 day, vomiting, drowsiness followed by seizures. On admission to PICU, she was drowsy, tachycardic, tachypneic, with rashes, and hepatosplenomegaly. Lab findings showed thrombocytopenia, leucopenia, low ESR, normal CRP, elevated liver enzymes, high ferritin, LDH, and triglycerides suggestive of macrophage activation syndrome (MAS). Chest X-ray showed left basal pneumonia and DNA PCR of throat swab revealed adenovirus. She was diagnosed as adenovirus-triggered MAS and was initiated on pulse methylprednisolone $(6 \mathrm{mg} / \mathrm{kg})$. Because of suboptimal response after 2 doses, manifested by increasing drowsiness, further fall in platelets and rising ferritin, methylprednisolone dosage was increased to $30 \mathrm{mg} / \mathrm{kg} /$ day with the addition of oral cyclosporine $(4 \mathrm{mg} / \mathrm{kg} / \mathrm{day})$. In view of worsening of the chest X-ray and increasing oxygen requirement, Cidofovir infusion $(1 \mathrm{mg} / \mathrm{kg}$ thrice weekly) was also started simultaneously considering increased activity of the adenoviral infection concurrent to immunosuppression. Within $48 \mathrm{~h}$, the child showed signs of recovery with improved consciousness, lower oxygen requirements, and improving lab parameters. She was discharged after 3 weeks of IV Cidofovir, on oral prednisolone and cyclosporine. To the best of our knowledge, this is the first reported use of Cidofovir in adenovirus-induced MAS.
\end{abstract}

Keywords Adenovirus · Cidofovir · Macrophage activation syndrome $\cdot$ Systemic arthritis

\section{Introduction}

Macrophage activation syndrome (MAS) is a potentially lethal complication of systemic inflammatory diseases of childhood, commonest association being with systemic juvenile idiopathic arthritis (sJIA). MAS may occur spontaneously,

Electronic supplementary material The online version of this article (https://doi.org/10.1007/s10067-020-05133-0) contains supplementary material, which is available to authorized users.

Priyankar Pal

mailme.priyankar@gmail.com

1 Institute of Child Health, 11, Dr Biresh Guha St., Kolkata 700017, India

2 Department of Pediatrics, Apollo Gleneagles Hospitals, Kolkata, India

3 Pediatric Intensive Care Unit, Apollo Gleneagles Hospitals, Kolkata, India as a complication of active underlying disease, or may be triggered by an infection, a change in drug therapy, or toxic effect of a medication. The acute and dramatic onset of this entity often mimics a flare of the underlying disease or a severe sepsis [1]. Adenoviruses are emerging as an important cause of respiratory tract infections and can lead to significant morbidity in the immunocompromised.

A 5-year-old girl, suffering from polycyclic sJA, presented with fever, poor oral intake, and drowsiness since morning. Her disease dates back to 18 months of age when she was first presented with fever and rash with elevated acute-phase reactants at another center and was initially diagnosed as Kawasaki disease that failed to respond to IVIg. She developed MAS and received pulse methylprednisolone. Subsequently on appearance of chronic arthritis, the diagnosis was revised to sJA. Over the last 3.5 years she had multiple systemic and articular flares and prior to this admission she was on fortnightly Tocilizumab $12 \mathrm{mg} / \mathrm{kg}$, weekly oral Methotrexate, Leflunomide and $0.3 \mathrm{mg} / \mathrm{kg}$ oral Prednisolone daily. She had received Tocilizumab 4 days prior to the present episode. 
By evening the parents reported increasing drowsiness, recurrent vomiting, and was advised for hospitalization. On their way to the hospital, she had one episode of generalized seizure lasting for few minutes that subsided spontaneously.

At presentation to the hospital, the child was irritable, pale, febrile $\left(101.1^{\circ} \mathrm{F}\right)$, moderately dehydrated, and hypoglycemic (CBS-27 mg\%) with erythematous maculopapular rashes over the trunk. She was tachycardic (180/min sinus), tachypneic (RR-52/min) with subcostal retractions, $\mathrm{SpO} 2$ of $87-89 \%$ at room air (90-92\% with face mask $4 \mathrm{~L} / \mathrm{min}$ ) with crepitations at left base. Abdominal examination revealed soft hepatomegaly with liver span of $9 \mathrm{~cm}$ and a just palpable spleen.

Initial labs showed a total white cell count of 2500 cells/ $\mathrm{mm}^{3}$ with polymorphonuclear cells being $30 \%$ and lymphocytes contributing $64 \%$ of total count. Hemoglobin was $10.2 \mathrm{~g} / \mathrm{dL}$, platelet count was $1.7 \mathrm{lac} / \mathrm{mm}^{3}$, ESR was $07 \mathrm{~mm}$, CRP was $1.9 \mathrm{mg} / \mathrm{dL}$ (cut-off $<0.5 \mathrm{mg} / \mathrm{dL}$ ), and procalcitonin was $4.9 \mathrm{ng} / \mathrm{dL}$ (cut-off $<0.5 \mathrm{mg} / \mathrm{dL}$ ). Liver enzymes were raised (SGOT-175 U/L, SGPT-322 U/L). INR was 1.26. ABG showed metabolic acidosis. Serum ferritin was $11,519 \mathrm{ng} / \mathrm{mL}$, serum triglycerides were $257 \mathrm{mg} / \mathrm{dl}$, serum LDH was $948 \mathrm{U} / \mathrm{L}$, and plasma fibrinogen was $221 \mathrm{mg} \%$.

Chest X-ray showed opacities in left lower zone. DNA PCR from nasopharyngeal swab revealed adenovirus.

Considering the clinical spectrum of fever with acute onset encephalopathy, hepatopathy, and cytopenias with low ESR in the background of sJIA, she was diagnosed as MAS in sJIA $[2,3]$ precipitated by an adenoviral infection and initiated on pulse methylprednisolone of $6 \mathrm{mg} / \mathrm{kg} /$ day along with broad spectrum antibiotics and other supportive measures. However, even after adequate fluid boluses and 2 doses of methylprednisolone, she remained acidotic (metabolic), drowsy with increasing work of breathing and increasing oxygen requirements. Repeat investigations after $24 \mathrm{~h}$ showed falling platelets $76,000 / \mathrm{mm} 3$, falling hemoglobin $7.9 \mathrm{~g} \%$, and rising ferritin $61,134 \mathrm{ng} / \mathrm{mL}$ with worsening chest X-ray (Fig. 1). Hence, a decision was taken to initiate Cidofovir with intensified immunosuppression (IV methylprednisolone pulse $30 \mathrm{mg} / \mathrm{kg}$ plus oral cyclosporine $4 \mathrm{mg} / \mathrm{kg} /$ day). Cidofovir was started at $1 \mathrm{mg} / \mathrm{kg}$ on alternate day, considering its nephrotoxic potential and concurrent use of cyclosporine. Within $48 \mathrm{~h}$ of the intensified treatment, the child was afebrile, with improved consciousness and decreasing oxygen requirement. Labs also improved, total count increased to $7200 / \mathrm{mm} 3$ with decreased transaminases, and ferritin drastically came down to $9034 \mathrm{ng} / \mathrm{mL}$, but platelet was $57,000 / \mathrm{mm} 3$. After 3 pulses of $30 \mathrm{mg} / \mathrm{kg}$, IV methylprednisolone was reduced to $6 \mathrm{mg} / \mathrm{kg} /$ dose for 2 days and on day 7 switched over to oral prednisolone $(2 \mathrm{mg} / \mathrm{kg} /$ day $)$. Her chest X-ray showed resolution of the pneumonia by day 7 (Fig. 2). However, considering the concurrent immunosuppression and the slow progressive potential of adenovirus, she was administered Cidofovir for 3 weeks with regular monitoring of renal functions.

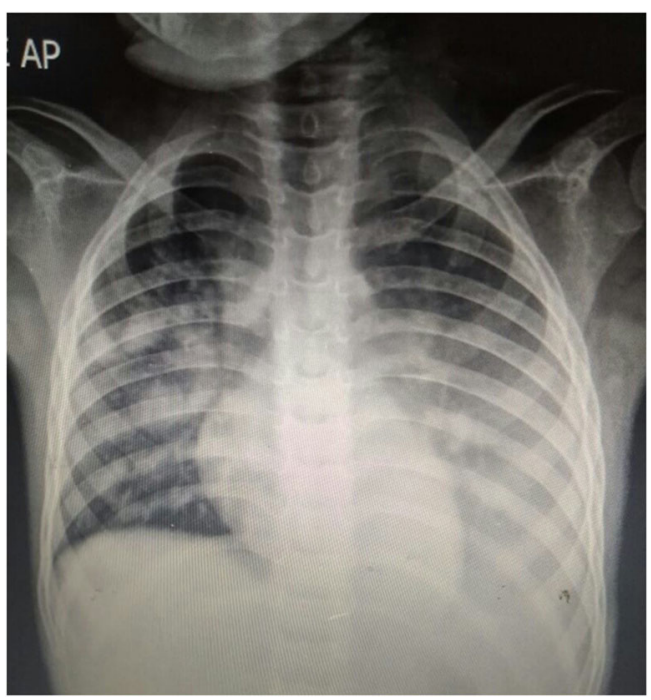

Fig. 1 Chest X-ray showing worsening bilateral pneumonia

At discharge, she had hemoglobin $11.2 \mathrm{~g} \%$, TLC $9000 / \mathrm{mm} 3$, platelet $2.51 \mathrm{lac} / \mathrm{mm} 3$, and ferritin $2069 \mathrm{ng} / \mathrm{mL}$. She remains well 6 months after follow-up.

\section{Discussion}

Adenoviruses are a family of DNA viruses known to cause febrile illnesses in young children. Though mostly self-limiting, they can be fatal in the immunocompromised and occasionally in healthy individuals. Viral culture remains the gold standard for diagnosis, but polymerase chain reaction based assays are highly sensitive and specific providing a rapid diagnosis. Multiplex PCR assays for detecting multiple

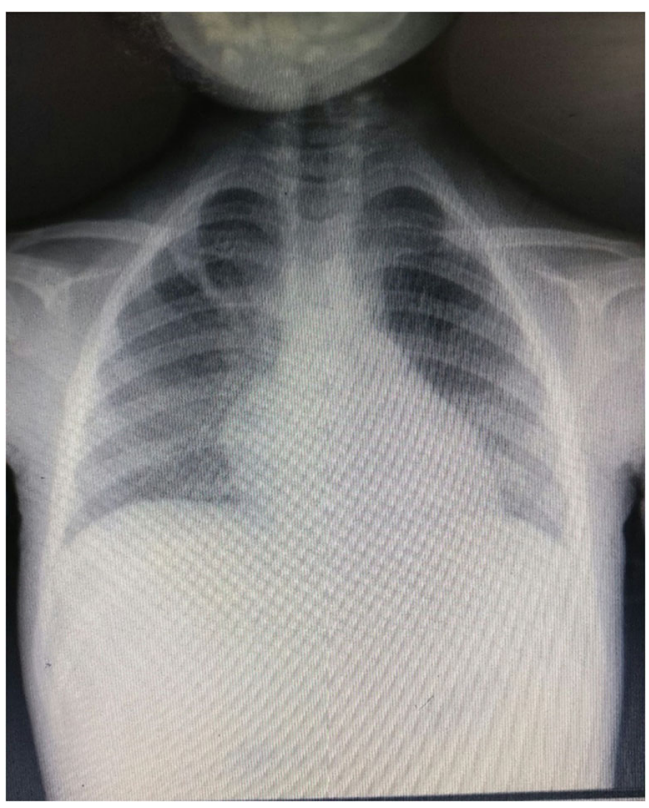

Fig. 2 Chest X-ray prior to discharge 
respiratory pathogens using nasopharyngeal secretions provide rapid diagnosis especially in hospitalized children for suspected pneumonia. Since detection of specific viruses by this method may represent colonization or shedding rather than infection, results need to be interpreted in the context of the clinical setting. Considering the clinical presentation of a febrile lower respiratory tract infection with worsening X-rays and adenovirus on nasopharyngeal swab, the child was diagnosed as adenoviral pneumonia.

Our patient had MAS at diagnosis of sJIA at the age of 18 months. Subsequently, she had multiple systemic and articular flares requiring polytherapy. However, the joints continued to remain partially resistant to all therapy with intermittent relapses. After her last dose of Tocilizumab $12 \mathrm{mg} / \mathrm{kg}$ at 4 days back, she had fever and vomiting for 1 day, and the parents reported increasing drowsiness which progressed to seizures. On admission, MAS was diagnosed on the basis of her clinical and biochemical parameters.

MAS is a potentially lethal complication of systemic inflammatory diseases of childhood, commonest association being with SJIA. The clinical features include unremittent fever, pancytopenia, hepatosplenomegaly, CNS symptoms, elevated liver enzymes, high ferritin, and low ESR. The pathogenesis is related to uncontrolled activation and proliferation of CD8 T lymphocytes and macrophages and this leads to over production of various cytokines resulting in a "cytokine storm" which is responsible for the varying manifestations.

It has been estimated that the prevalence of MAS among patients with sJIA is around $10 \%$, but current data from Behrens et al. and Blessing et al. suggest that subclinical MAS may occur even in 30-40\% of patients with sJIA [4, 5].

Davi et al. [6] in their randomized multi-centric study of 362 patients with sJIA-associated MAS found that MAS was present in $22 \%$ cases of sJIA at onset. Significant features of MAS included fever (96\%, often persistent rather than quotidian patterns), hepatomegaly (70\%), splenomegaly (58\%), and lymphadenopathy (51\%). Central nervous system dysfunction ranging from seizures, lethargy, irritability, confusion, headache, and coma occurred in 35\%. Twenty-six percent of patients had cardiac involvement with pericardial involvement being the most common. Pulmonary involvement with pleural effusions occurred in $22 \%$ of patients. Bleeding manifestations ranged from petechiae, purpura, mucosal, or gastrointestinal bleeding in $20 \%$ and disseminated intravascular coagulation in $1 \%$. Renal involvement ranging from renal failure, hematuria, and proteinuria was seen in $15 \%$. It is prudent to note that MAS was present in almost $40 \%$ of patients who did not demonstrate tissue hemophagocytosis. Laboratory features of MAS include a drop in ESR, WBC, platelet counts, and fibrinogen levels with extremely elevated ferritin levels, elevated CRP and liver enzymes, lactate dehydrogenase, triglycerides, D-dimer, and prolonged prothrombin time and partial thromboplastin time. Soluble IL-2 receptor and soluble
CD163 are also elevated in active MAS [7]. The low CRP of the patient at admission in the setting of a MAS was attributed to the recent Tocilizumab administration [8], rise in CRP being IL6 mediated.

The child was initiated on $6 \mathrm{mg} / \mathrm{kg}$ pulse methylprednisolone but continued to deteriorate even after 2 doses with increasing respiratory distress along with worsening of X-ray and laboratory parameters. Thus, a decision was made to increase immunosuppression, with concurrent administration of the antiviral Cidofovir to counter the possible expected viremic flare.

Cidofovir (CDV) was the first nucleotide analogue approved by the FDA for clinical use in 1996 for treating cytomegalovirus retinitis in AIDS patients. It shows in vitro activity against a number of DNA viruses including adenovirus, herpes, pox, polyoma, and papilloma viruses. Though rigorously used clinically in AIDS patients for CMV retinitis treatment, it was subsequently found to be more active than other antivirals for treating adenoviral infections and has been used for treatment of invasive adenoviral infections in immunecompromised patients. However, published data on the efficacy of CDV for adenovirus infection are limited to case reports and small nonrandomized series $[9,10]$. In hematopoietic stem cell and lung transplant recipients, early administration of CDV has been associated with decreased viral load, clinical improvement, and increased survival [10-13]. Ganapathi et al. [14] in their study in 2016 showed that CDV was associated with decreased viral response and clinical improvement in the majority of patients.

Dose-dependent nephrotoxicity remains a serious concern with use of Cidofovir and is contraindicated in patients with significant proteinuria and a baseline creatinine of more than $1.5 \mathrm{mg} / \mathrm{dL}$. However, using $1 \mathrm{mg} / \mathrm{kg}$ every alternate day with hydration and concurrent use of probenecid has been shown to reduce renal toxicity [15]. Considering the concurrent usage with cyclosporine, we used the thrice weekly regimen along with probenecid with strict monitoring of renal functions.

With $30 \mathrm{mg} / \mathrm{kg}$ pulse methylprednisolone plus oral cyclosporine and Cidofovir, the child showed steady improvement. The optimum duration of Cidofovir therapy is not standardized but has to be individualized depending on therapeutic response. Since our patient was on long-term immunosuppressives and was planned discharge on oral Prednisolone and cyclosporine, we decided to administer Cidofovir for a total of 3 weeks duration.

Infection remains an important precipitant of MAS and the cytokine storm syndrome, the COVID-19 current pandemic being a classical example [16-18]. Adenovirus is also emerging as an important respiratory pathogen that can lead to serious morbidity in immune competent and mortality in the immunocompromised. Early identification of the pathogen and timely initiation of Cidofovir in select individuals have been shown to improve consequences, though currently use of the 
drug is limited to hemato-oncologists in transplant patients. To the best of our knowledge, this is the first reported usage of Cidofovir as an antiviral in MAS induced by an adenoviral infection.

\section{Compliance with ethical standards}

Disclosures None.

\section{References}

1. Grevich S, Shenoi S (2017) Update on the management of systemic juvenile idiopathic arthritis and role of IL-1 and IL-6 inhibition. Adolesc Health Med Ther 8:125-135

2. Ravelli A, Minoia F, Davi S et al (2016) 2016 classification criteria for macrophage activation syndrome complicating systemic juvenile idiopathic arthritis: a European league against rheumatism/ American College of Rheumatology/Paediatric Rheumatology International Trials Organisation Collaborative Initiative. Ann Rheum Dis 75(3):481-489

3. Minoia F, Davi S, Horne A, Demirkaya E, Bovis F, Caifeng L, on behalf of the Pediatric Rheumatology International Trials Organization, the Childhood Arthritis and Rheumatology Research Alliance, the Pediatric Rheumatology Collaborative Study Group, and the Histiocyte Society et al (2014) Clinical features, treatment, and outcome of macrophage activation syndrome complicating systemic juvenile idiopathic arthritis: a multinational, multicenter study of 362 patients. Arthritis Rheumatol 66:31603169

4. Behrens EM, Beukelman T, Paessler M, Cron RQ (2007) Occult macrophage activation syndrome in patients with systemic juvenile idiopathic arthritis. J Rheumatol 34:1133-1138

5. Bleesing J, Prada A, Siegel DM, Villanueva J, Olson J, Ilowite NT, Brunner HI, Griffin T, Graham TB, Sherry DD, Passo MH, Ramanan AV, Filipovich A, Grom AA (2007) The diagnostic significance of soluble CD163 and soluble interleukin-2 receptor achain in macrophage activation syndrome and untreated new-onset systemic juvenile idiopathic arthritis. Arthritis Rheum 56:965-971

6. Minoia F, Davi S, Horne A et al (2014) Clinical features, treatment, and outcome of macrophage activation syndrome complicating systemic juvenile idiopathic arthritis: a multinational, multicenter study of 362 patients. Arthritis Rheumatol 66(11):3160-3169

7. Cimaz R (2016) Systemic-onset juvenile idiopathic arthritis. Autoimmun Rev 15(9):931-934
8. Schulert GS, Minoia F, Bohnsack J, Cron RQ, Hashad S, KonÉPaut I et al Biologic therapy modifies clinical and laboratory features of macrophage activation syndrome associated with systemic juvenile idiopathic arthritis. Arthritis Care Res 70(3):409-419

9. Leruez-Ville M, Minard V, Lacaille F, Buzyn A, Abachin E, Blanche S, Freymuth F, Rouzioux C (2004) Real time blood polymerase chain reaction for management of disseminated adenovirus infection. Clin Infect Dis 38:45-52

10. Neofytos D, Ojha A, Mookerjee B, Wagner J, Filicko J, Ferber A, Dessain S, Grosso D, Brunner J, Flomenberg N, Flomenberg P (2007) Treatment of adenovirus disease in stem cell transplant recipients with cidofovir. Biol Blood Marrow Transplant 13:74-81

11. Legrand F, Berrebi D, Houhou N et al (2001) Early diagnosis of adenovirus infection and treatment with cidofovir after bone marrow transplantation in children. Bone Marrow Transplant 27:261

12. Ljungman P, Ribaud P, Eyrich $M$ et al (2003) Cidofovir for adenovirus infections after allogenic hematopoietic stem cell transplantation: a survey by the infectious diseases working Party of the European Group for blood and marrow transplantation. Bone Marrow Transplant 31-481

13. Doan ML, Mallory GB, Kaplan SL, Dishop MK, Schecter MG, McKenzie ED, Heinle JS, Elidemir O (2007) Treatment of adenovirus pneumonia with cidofovir in pediatric lung transplant recipients. J Heart Lung Transplant 26:883-889

14. Ganapathi L, Arnold A, Jones S et al (2016) Use of cidofovir in pediatric patients with adenovirus infection. F1000Res 5:758. Published 2016 Dec 16. https://doi.org/10.12688/f1000research. 8374.2

15. Vora SB, Brothers AW, Englund JA (2017) Renal toxicity in pediatric patients receiving cidofovir for the treatment of adenovirus infection. J Pediatric Infect Dis Soc 6(4):399-402

16. Sarzi-Puttini P, Giorgi V, Sirotti S, Marotto D, Ardizzone S, Rizzardini G, Antinori S, Galli M (2020) COVID-19, cytokines and immunosuppression: what can we learn from severe acute respiratory syndrome? Clin Exp Rheumatol 38:337-342

17. Chi Zhang MD, Zhao Wu PD, Jia-Wen Li MD et al (2020) The cytokine release syndrome ( CRS) of severe COVID-19 and Interleukin-6 receptor antagonist Tocilizumab may be the key to reduce mortality. Int J Antimicrob Agents 105954. https://doi.org/ 10.1016/j.ijantimicag.2020.105954

18. Gupta L, Agarwal V, Ramanan AV. Interleukin -6 and other cytokine blockade in COVID-19 hyperinflammation. Indian J Rheumatol 0;0:0

Publisher's note Springer Nature remains neutral with regard to jurisdictional claims in published maps and institutional affiliations. 